\title{
Expression of interferon-regulated genes in juvenile dermatomyositis versus Mendelian autoinflammatory interferonopathies
}

\author{
Hanna Kim, Fatima Gunter-Rahman², John A. McGrath ${ }^{3}$, Esther Lee ${ }^{2}$, Adriana A. de Jesus ${ }^{4}$, Ira N. Targoff ${ }^{5}$, \\ Yan Huang ${ }^{4}$, Terrance P. O'Hanlon'², Wanxia L. Tsai ${ }^{6}$, Massimo Gadina ${ }^{6}$, Frederick W. Miller' ${ }^{2}$, \\ Raphaela Goldbach-Mansky ${ }^{4+}$ and Lisa G. Rider ${ }^{2+}$
}

\begin{abstract}
Background: Juvenile dermatomyositis (JDM) is a systemic autoimmune disease with a prominent interferon (IFN) signature, but the pathogenesis of JDM and the etiology of its IFN signature remain unknown. The Mendelian autoinflammatory interferonopathies, Chronic Atypical Neutrophilic Dermatosis with Lipodystrophy and Elevated temperature (CANDLE) and STING-Associated Vasculopathy with onset in Infancy (SAVI), are caused by genetic mutations and have extremely elevated IFN signatures thought to drive pathology. The phenotypic overlap of some clinical features of CANDLE and SAVI with JDM led to the comparison of a standardized interferon-regulated gene score (IRG-S) in JDM and myositis-specific autoantibody (MSA) JDM subgroups, with CANDLE and SAVI.

Methods: A peripheral 28-component IRG-S assessed by NanoString ${ }^{\text {TM }}$ in 57 JDM patients subtyped by MSA was compared with IRG-S in healthy controls (HC) and CANDLE/SAVI patients. Principal component analysis (PCA) was performed, and individual genes were evaluated for their contribution to the score. IRG-S were correlated with disease assessments and patient characteristics.

Results: IRG-S in JDM patients were significantly higher than in HC but lower than in CANDLE or SAVI. JDM IRG-S overlapped more with SAVI than CANDLE by PCA. Among MSA groups, anti-MDA5 autoantibody-positive patients' IRG-S overlapped most with SAVI. The IFI27 proportion was significantly higher in SAVI and CANDLE than JDM, but IFIT1 contributed more to IRG-S in JDM. Overall, the contribution of individual interferon-regulated genes (IRG) in JDM was more similar to SAVI. IRG-S correlated moderately with JDM disease activity measures $\left(r_{s}=0.33-0.47\right)$ and more strongly with skin activity $\left(r_{s}=0.58-0.79\right)$ in anti-TIF1 autoantibody-positive patients. Weakness and joint disease activity (multinomial OR 0.91 and 3.3) were the best predictors of high IRG-S.

\footnotetext{
* Correspondence: hanna.kim@nih.gov

${ }^{\dagger}$ Raphaela Goldbach-Mansky and Lisa G. Rider contributed equally to this work.

${ }^{1}$ Pediatric Translational Research Branch, National Institute of Arthritis and Musculoskeletal and Skin Diseases, NIH, Bethesda, MD, USA

Full list of author information is available at the end of the article
}

(c) The Author(s). 2020 Open Access This article is licensed under a Creative Commons Attribution 4.0 International License, which permits use, sharing, adaptation, distribution and reproduction in any medium or format, as long as you give appropriate credit to the original author(s) and the source, provide a link to the Creative Commons licence, and indicate if changes were made. The images or other third party material in this article are included in the article's Creative Commons licence, unless indicated otherwise in a credit line to the material. If material is not included in the article's Creative Commons licence and your intended use is not permitted by statutory regulation or exceeds the permitted use, you will need to obtain permission directly from the copyright holder. To view a copy of this licence, visit http://creativecommons.org/licenses/by/4.0/ The Creative Commons Public Domain Dedication waiver (http://creativecommons.org/publicdomain/zero/1.0/) applies to the data made available in this article, unless otherwise stated in a credit line to the data. 


\begin{abstract}
(Continued from previous page)
Conclusions: Our findings demonstrate peripheral IRG expression in JDM overlaps with monogenic interferonopathies, particularly SAVI, and correlates with disease activity. Anti-MDA5 autoantibody-positive JDM IRG-S were notably more similar to SAVI. This may reflect both a shared IFN signature, which is driven by IFN- $\beta$ and STING pathways in SAVI, as well as the shared phenotype of vasculopathy in SAVI and JDM, particularly in anti-MDA5 autoantibody-positive JDM, and indicate potential therapeutic targets for JDM.
\end{abstract}

Keywords: (3-10): juvenile dermatomyositis, Myositis, Pediatric rheumatology, Interferon, Biomarkers, Interferonopathy, Myositis-specific autoantibodies

\section{Background}

Juvenile dermatomyositis (JDM) is a complex autoimmune disease characterized by weakness and rashes [1]. Myositis-specific autoantibodies (MSA) define phenotypic features and prognosis within JDM, with anti-TIF1, antiNXP2, and anti-MDA5 autoantibodies being the most common MSA groups in JDM [1-4]. Although the etiology of JDM is presently unknown, multiple genetic and environmental factors contribute $[1,5]$. Interferon (IFN)regulated genes (IRG) are upregulated in the blood, muscle, and skin of patients with JDM and adult dermatomyositis (DM) and correlate with disease activity [6-13], although the exact source, mechanism, and role of IFN remain unclear and detailed assessments by MSA group are lacking.

Mendelian autoinflammatory interferonopathies, which are associated with a strong IRG signature, include Chronic Atypical Neutrophilic Dermatosis with Lipodystrophy and Elevated temperature (CANDLE) caused by additive loss-of-function mutations in proteasome components [14-16] and STING-Associated Vasculopathy with onset during Infancy (SAVI), resulting from gain-offunction mutations in the Stimulator of IFN genes (STING) protein [16, 17]. These conditions have a very strong IRG signature and blocking IFN signaling with a Janus kinase (JAK) inhibitor correlates with clinical improvement and IRG-S decrease in the majority of 18 CANDLE and SAVI patients, with 50\% of CANDLE patients achieving persistent clinical remission [16, 18-20]. A direct comparison of JDM to the monogenic interferonopathies may provide insight into the role of IFN in JDM, particularly as CANDLE and SAVI share some clinical features with JDM. For example, CANDLE is also associated with lipodystrophy, joint contractures, and myositis, while SAVI has frequent vasculopathy including distal ulcerations and interstitial lung disease $[1,16,21]$.

Utilizing a 28 IRG score (IRG-S), which was developed and validated as a biomarker in CANDLE and SAVI [22], we evaluated peripheral blood IRG expression in JDM. Given prominent IRG signatures and overlapping clinical features of JDM with conditions with IFN-driven pathogenesis based on genetic mutations (CANDLE and SAVI), we aimed to better characterize and understand the role of IFN in JDM and its MSA subgroups through direct comparison to patients with CANDLE and SAVI.

\section{Methods \\ Patient selection}

Subjects were enrolled in National Institutes of Health institutional review board-approved natural history studies (Table 1). Active JDM patients $(n=57)$ met probable or definite Bohan and Peter criteria [23]. CANDLE $(n=11)$ and SAVI $(n=7)$ patients were genetically defined and used as positive interferonopathy controls [18]. Neonatalonset multisystem inflammatory disease (NOMID) patients $(n=18)$ served as autoinflammatory controls (IL-1 mediated), in addition to 26 healthy controls (HC), both of which were IFN-negative [22]. $\mathrm{HC}$ were not age- or gender-matched to JDM patients, as we previously did not find significant differences in IRG-S based on these variables [22]. All JDM patients consented to a NIH/NIEHS IRB-approved protocol. All CANDLE, SAVI, NOMID, and HC patients consented to a NIH/NIAID IRBapproved protocol (NCT02974595). MSAs were identified by validated immunoprecipitation and immunoblotting methods [24]. MSAs with adequate numbers of JDM patients for analysis included anti-p155/140 (TIF1) $(n=21)$, anti-MJ (NXP2) $(n=11)$, anti-MDA5 $(n=11)$ autoantibodies, and MSA-negative $(n=9)$. Clinical data are available to characterize features for 56 JDM patients.

\section{Materials}

From a single sample per patient of whole blood collected in PAXgene tubes (Qiagen, Germantown, MD), total RNA was extracted. NanoString Technologies ${ }^{\text {Tw }}$ (Seattle, WA) was used for gene expression analysis, with scores calculated as a sum of $Z$-scores for each of 28 IRGs [22]. Exploratory analysis (data not shown) of complete blood count parameters (white blood cell count as well as absolute neutrophil, lymphocyte, monocyte, eosinophil, and basophil counts) between JDM $(n=55)$ versus CANDLE $(n=10)$ and SAVI $(n=5)$ FDR corrected for multiple comparisons did not detect any significant differences, so no further normalization based on these counts was performed. 
Table 1 Demographics of each condition

\begin{tabular}{|c|c|c|c|c|c|c|}
\hline \multirow{2}{*}{\multicolumn{2}{|c|}{ Characteristics }} & \multicolumn{5}{|c|}{ Median [IQR] or $N(\%)$} \\
\hline & & $\operatorname{JDM}(n=57)$ & CANDLE $(n=11)$ & SAVI $(n=7)$ & $\operatorname{NOMID}(n=18)$ & $\mathrm{HC}(n=26)$ \\
\hline \multicolumn{2}{|c|}{ Age at evaluation (year) } & $9.5[5.9-13.2]$ & $6.1[5.2-15.7]$ & $16.9[6.1-18.1]$ & $8.5[5.3-17.1]$ & $25.9[9.7-39.4]$ \\
\hline Gender & Female & $34(60)$ & $5(45)$ & $3(43)$ & $7(39)$ & $17(65)$ \\
\hline \multirow[t]{5}{*}{ Race } & White & $36(63)$ & $4(36)$ & $7(100)$ & $12(67)$ & $15(58)$ \\
\hline & Hispanic & $4(7)$ & $4(36)$ & 0 & $3(17)$ & $8(31)$ \\
\hline & Black & $3(5)$ & $2(18)$ & 0 & $1(6)$ & $1(4)$ \\
\hline & Multiple race & $10(18)$ & 0 & 0 & $1(6)$ & $1(4)$ \\
\hline & Other & $4(7)$ & $1(9)$ & 0 & $1(6)$ & $1(4)$ \\
\hline \multicolumn{2}{|c|}{ Disease duration (months) } & $10.7[4.9-36.7]$ & NA & NA & NA & NA \\
\hline
\end{tabular}

Abbreviations: IQR interquartile range, NA not applicable, JDM juvenile dermatomyositis, CANDLE Chronic Atypical Neutrophilic Dermatosis with Elevated temperature, SAVI STING-Associated Vasculopathy with onset during Infancy, NOMID Neonatal-onset multisystem inflammatory disease, HC healthy controls

\section{Analysis methods}

Analysis was performed using $\mathrm{R}$ version 3.5.0 (The $\mathrm{R}$ Foundation for Statistical Computing, Vienna, Austria, ISBN 3-9000-51-07-0, http://www.r-project.org), GraphPad Prism7 (GraphPad Software, San Diego, CA), JMP13 (SAS Institute, Cary, NC), or SYSTAT13 (Systat Software, San Jose, CA).

\section{Whole blood score comparisons}

With JDM overall, IRG-S were compared to CANDLE, SAVI, NOMID, and HC, and FDR corrected for multiple comparisons using the Benjamini, Krieger, and Yekutieli method [25], with significant $q$ values $<0.05$. Exploratory analysis compared subgroups of JDM (highest quartile of JDM IRG-S (JDM-HQ), JDM IRG-S above HC, or a specific MSA group) to each other, autoinflammatory conditions and/or HC by Kruskal-Wallis tests, followed by Dunn's multiple comparisons tests (uncorrected), with $p<0.05$ considered significant.

\section{Principal component analysis}

For principal component analysis (PCA) of JDM, JDM$\mathrm{HQ}$, and MSA subgroups with the autoinflammatory diseases and controls, all PCAs had adequate samples, as indicated by Kaiser-Meyer Olkin test (values $\geq 0.86$ ) and Bartlett's sphericity test ( $p$ values $<0.0001)$. Five unrotated PCAs were performed using normalized gene counts, each with autoinflammatory diseases and $\mathrm{HC}$ data, differentiated by whether they included all JDM patients or a MSA subgroup as follows: PCA-A (all JDM, CANDLE, SAVI, NOMID, HC), PCA-B (anti-MDA5 autoantibody-positive subgroup of JDM, CANDLE, SAVI, NOMID, HC), PCA-C (anti-NXP2 autoantibodypositive subgroup of JDM, CANDLE, SAVI, NOMID, HC), PCA-D (anti-TIF1 autoantibody-positive subgroup of JDM, CANDLE, SAVI, NOMID, HC), and PCA-E (MSA-negative subgroup of JDM, CANDLE, SAVI, NOMID, HC). One anti-TIF1 JDM patient with suspected viral bronchitis had outlying IRG expression (>99th percentile) and principal component (PC) scores, and was removed from the analysis. The component loadings are the correlations of the individual gene normalized $Z$ scores with the given principal component. A stronger loading (e.g., greater than 0.40 or less than -0.40 ) indicates a stronger relationship or contribution of that gene with that principal component. As with correlations, these can be negative or positive.

\section{Gene proportion analysis}

The contribution of each of the 28 individual IRGs was compared to the total IRG Z-score, which was normalized so that no gene component was negative. Each gene's proportion of the total normalized IRG-S in JDMHQ was compared to that in CANDLE and SAVI. The analysis was limited to JDM-HQ $(n=14)$; this subgroup of JDM has an IRG-S in the same range as CANDLE and SAVI for a more equitable comparison of individual gene proportions. As our goal was to assess whether the pattern of dysregulation in JDM IRG-S was the same as in CANDLE and SAVI, we assessed gene proportions from scores in the same IRG-S range in order to limit differences due simply to different levels of overall dysregulation.

\section{Ratios related to NF- $\mathrm{KB}$ and IFN- $\gamma$}

The expression level of the 3 IFN response genes (CXCL10, GPB1, SOCS1) that are regulated by STAT1 and have nuclear factor kappa $\mathrm{B}(\mathrm{NF}-\kappa \mathrm{B})$ binding sites are differentially regulated in some conditions with elevated IRG-S, distinguishing a subset of diseases from CANDLE and SAVI [26]. Also, as the contribution of different interferons can be variable, the expression level of 9 of the IFN response genes (CXCL10, EPSTI1, IFIT1, IFIT2, IFIT3, ISG15, LY6E, SOCS1, USP18) with a greater increase in expression when induced by IFN- $\gamma$ than IFN- $\alpha$, over all 28 genes, was assessed as this has also been described to be variable in other interferonopathies versus CANDLE and SAVI [26]. Both the ratio 
of 3 NF-kB-related genes compared to the other 25 IRGs ("NF- $\mathrm{kB}$ ratio") and the latter ratio of 9 genes over 28 ("IFN- $\gamma$ ratio") [26] were calculated based on normalized counts with analysis as above in JDM patients with elevated scores or IRG-S above healthy control range [22] and described by MSA group versus CANDLE and SAVI.

\section{IFN score correlations in JDM}

IRG-S were correlated by Spearman's rank $\left(r_{s}\right)$ with core set disease activity and damage assessments in JDM patients overall and in patients with anti-TIF1 autoantibodies [27] (Table 2). IRG-S were also correlated with corticosteroid doses. IRG-S was compared between JDM patients receiving specific medications or not by the
Mann-Whitney test and analyzed by the number of steroid-sparing medications received via Kruskal-Wallis and Dunn's (uncorrected) tests.

Two published IRG-S from DM patients, IFIT1, IRF7, and ISG15 [6] and EPSTI1, HERC5, IFI27, IFI44, IFI44L, IFI6, IFIT1, IFIT3, ISG15, MX1, OAS1, OAS3, and RSAD2 [7], were calculated by summing the $Z$-score of relevant genes for correlation with JDM disease assessments.

JDM disease activity and damage core measures [27] (Additional file 1: Table S1) were compared among IRG-S above and below 48.9, the 95th percentile of $\mathrm{HC}$ [22], using Mann-Whitney or Fisher's exact tests. Myositis Disease Activity Assessment Tool (MDAAT) cardiovascular and gastrointestinal visual analog scales (VAS) were excluded, as few patients had activity in these systems. Non-

Table 2 Spearman's rank correlations between selected myositis disease measures and interferon-regulated gene (IRG) scores

\begin{tabular}{|c|c|c|c|c|c|}
\hline \multirow[b]{2}{*}{$\begin{array}{l}\text { Assessment }(n=57) \text { (range or upper } \\
\text { limit of normal }(U L N))\end{array}$} & \multirow[b]{2}{*}{ JDM overall median, IQR } & \multicolumn{2}{|c|}{28 gene IRG score } & \multicolumn{2}{|c|}{ Other IRG scores in JDM } \\
\hline & & $\begin{array}{l}\text { JDM overall } \\
(n=57)\end{array}$ & $\begin{array}{l}\text { Anti-TIF1 Ab } \\
(n=20)^{*}\end{array}$ & Bilgic et al. score ${ }^{\ddagger}[6]$ & Greenberg et al. score ${ }^{\S}[7]$ \\
\hline 28 IRG score $(n=57)$ & $40.0[1.8-236.0]$ & $40.0[1.8-236.0]$ & $41.7[-4.4-174.2]$ & $0.96^{* *}$ & $0.99^{* *}$ \\
\hline $\begin{array}{l}\text { Physician global activity (0-10 VAS, } \\
n=56 \text { ) }\end{array}$ & $2.2[1.5-3.7]$ & $0.39 \|$ & 0.31 & $0.42^{\|}$ & $0.39^{\|}$ \\
\hline Total MMT $(0-260, n=36)$ & $242[228-252]$ & $-0.36^{\S}$ & -0.13 & $-0.38^{\S}$ & -0.33 \\
\hline CMAS $(0-52, n=38)$ & $43[37-48]$ & -0.26 & -0.22 & -0.21 & -0.18 \\
\hline CHAQ/HAQ $(0-3.0, n=42)$ & $0.75[0.25-1.375]$ & 0.28 & 0.23 & 0.24 & 0.30 \\
\hline \multicolumn{6}{|l|}{ MDAAT (0-10 VAS, $n=39)$} \\
\hline Muscle & $2.0[1.0-2.9]$ & 0.31 & 0.29 & $0.40^{\S}$ & 0.30 \\
\hline Constitutional & $1.3[0.3-3.3]$ & 0.09 & 0.21 & 0.09 & 0.11 \\
\hline Cutaneous & $2.4[1.1-3.8]$ & 0.29 & $0.62^{\S}$ & $0.33^{\S}$ & 0.30 \\
\hline Pulmonary & $0.6[0.0-1.4]$ & 0.23 & 0.44 & 0.29 & 0.21 \\
\hline Skeletal (joint) & $0.9[0.0-2.1]$ & $0.35^{\S}$ & -0.12 & $0.42^{\|}$ & $0.36^{\S}$ \\
\hline Extra-muscular activity & $1.9[1.2-3.2]$ & $0.47^{\|}$ & $0.76^{\|}$ & $0.53^{n}$ & $0.48^{\|}$ \\
\hline \multicolumn{6}{|l|}{$(0-10$ VAS, $n=40)$} \\
\hline Disease Activity Score $(0-20, n=39)$ & 11 [9-13] & $0.33^{\S}$ & $0.58^{\S}$ & $0.35 \S$ & $0.33^{\S}$ \\
\hline DAS skin $(0-9, n=40)$ & $6[5-7]$ & 0.16 & $0.73^{\|}$ & 0.18 & 0.19 \\
\hline DAS muscle $(0-11, n=39)$ & $5[3.75-7]$ & 0.25 & 0.24 & 0.26 & 0.23 \\
\hline \multicolumn{6}{|l|}{ Muscle enzymes (U/L) } \\
\hline CK (ULN 252 U/L, $n=56)$ & $90[52-170]$ & -0.09 & -0.18 & -0.11 & -0.07 \\
\hline Aldolase (ULN $7 \mathrm{U} / \mathrm{L}, n=54)$ & $7.5[5.8-10.1]$ & 0.24 & 0.18 & 0.25 & 0.24 \\
\hline AST (ULN 34 U/L, $n=56)$ & 24 [18-33] & $0.42^{\|}$ & 0.24 & $0.38^{\|}$ & $0.43^{n}$ \\
\hline LDH (ULN 226 U/L, $n=56)$ & $186[161-214]$ & $0.41^{\|}$ & 0.27 & $0.39^{\|}$ & $0.39^{\|}$ \\
\hline $\begin{array}{l}\text { Physician global damage (0-10, } \\
n=46)\end{array}$ & $1.0[0.4-2.0]$ & -0.05 & 0.04 & -0.09 & -0.05 \\
\hline MDI total damage $(0-110, n=34)$ & $4.0[1.8-8.0]$ & 0.16 & 0.37 & 0.15 & 0.17 \\
\hline
\end{tabular}

Abbreviations: IQR interquartile range, JDM juvenile dermatomyositis, anti-TIF1 subgroup of JDM positive for anti-TIF1 autoantibodies, Ab autoantibody, VAS visual analog scale, MMT manual muscle testing, CMAS Childhood Myositis Assessment Scale, CHAQ: Childhood Health Assessment Questionnaire, HAQ Health

Assessment Questionnaire, MDAAT Myositis Disease Activity Assessment Tool, DAS Disease Activity Score, CK creatine kinase, AST aspartate aminotransferase, LDH lactate dehydrogenase, $P G D$ Physician global damage, $M D I$ Myositis Damage Index

${ }^{\S} p \leq 0.05, " \| 0.01,{ }^{\natural} p \leq 0.001,{ }^{* *} p \leq 0.0001$

*Proportionate numbers had assessments as JDM overall

${ }^{\dagger}$ Bilgic et al. score: IFIT1, IRF7, and ISG15 as in [6]

${ }^{\ddagger}$ Greenberg et al. score: EPSTI1, HERC5, IFI27, IFI44, IFI44L, IFI6, IFIT1, IFIT3, ISG15, MX1, OAS1, OAS3, and RSAD2 as in [7] 
redundant measures $\left(r_{s}<0.7\right)$ that differentiated high and low IRG-S were included in backward stepwise logistic regression to examine those associated (alpha $<0.15$ ) with high IRG-S (> 48.9).

\section{Interferon-related protein correlation}

IP-10 (CXCL10) as part of a Luminex assay (Bio-Rad, Hercules, CA) was assessed in serum or plasma of JDM patients available, to compare the IRG-S with IFNsignaling at the protein level by Spearman's correlation.

\section{Results}

\section{Description of clinical features}

Clinical characteristics were also descriptively compared between disease groups based on medical record review. Our cohort of JDM patients demonstrates some clinical overlap with CANDLE and SAVI including the presence of myositis, certain cutaneous features, musculoskeletal and systemic features, and damage findings albeit with different frequencies (Additional file 1: Table S2). Cutaneous features have distinctive characteristics in each condition, specifically heliotrope, Gottron's papules, photosensitivity, V-sign, and shawl sign rashes present in JDM, whereas panniculitis, nodular violaceous erythema, and annular plaques are present prominently in CANDLE, and cutaneous ulceration in SAVI. All of the JDM patients had proximal weakness while $8 / 10(80 \%)$ of CANDLE and $1 / 4$ (25\%) of SAVI had myositis. All of the SAVI patients with available data $(4 / 4,100 \%)$ had interstitial lung disease versus $8 / 56(14.3 \%)$ of JDM and none of the CANDLE patients. Of note, there is some variation within JDM by the MSA group as previously described [1-4]. The anti-TIF1 autoantibody JDM subgroup has more photosensitivity $(18 / 20,90.0 \%)$ and shawl sign rash $(8 / 20,55.0 \%)$ versus other groups in JDM. The anti-NXP2 autoantibody JDM subgroup has more notable myositis with MMT $<225 / 260$ $(2 / 5,40 \%)$ and less photosensitivity $(5 / 11,45.5 \%), \mathrm{V}$-sign $(1 / 11,9.1 \%)$, and shawl sign $(0 / 11,0.0 \%)$. Both have less interstitial disease $(0 / 20$ and $0 / 11,0.0 \%)$. The anti-MDA5 autoantibody JDM subgroup has less notable myositis with MMT <225/260 (0/8, 0.0\%) and more cutaneous ulceration and interstitial lung disease (both 7/10, 70.0\%) (Additional file 1: Table S2).

\section{Whole blood IRG score analysis}

JDM patients (median 40) had higher 28 IRG-S than patients with NOMID and HC and lower IRG-S than CANDLE and SAVI patients (median 534 and 501, respectively). IRG-S of JDM-HQ patients (median 473) did not differ from CANDLE and SAVI patients (Fig. 1). The IRG-S for each of the MSA groups were higher than in patients with NOMID and HC and did not differ from each other. IRG-S of JDM patients with anti-TIF1 and anti-NXP2 autoantibodies (median 42 and 25, respectively) were lower than

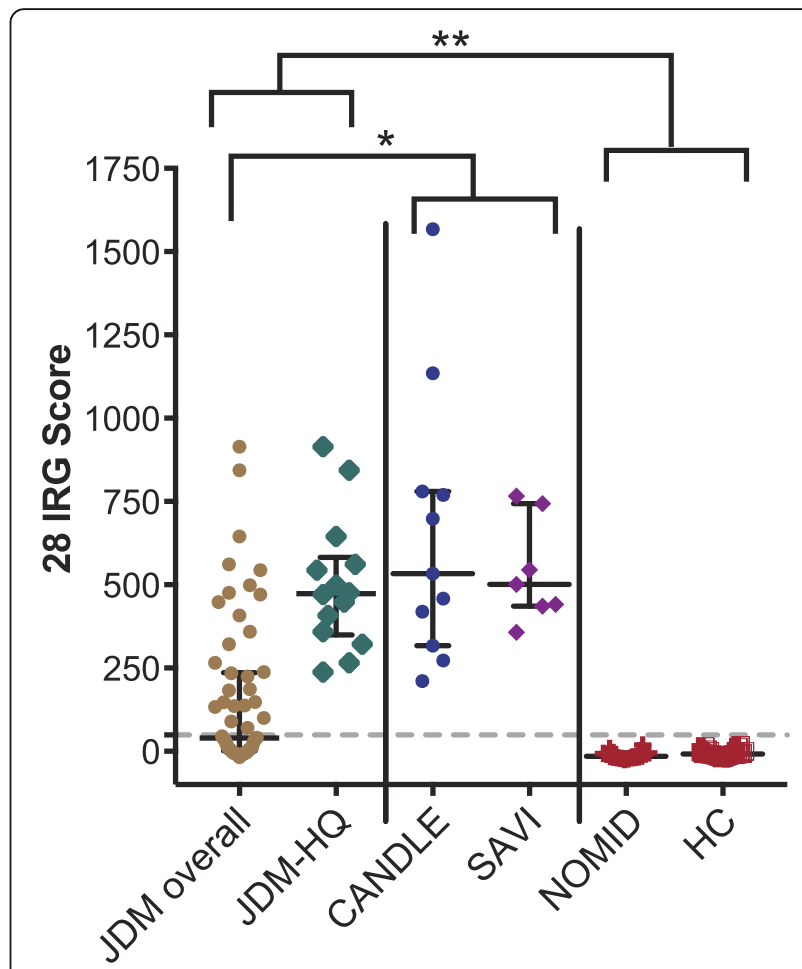

Fig. 1 Peripheral blood interferon-regulated gene (IRG) scores in juvenile dermatomyositis (JDM) compared to monogenic autoinflammatory disease patients and healthy controls. Median and interquartile ranges are shown. Dotted horizontal line represents 95th percentile of healthy controls [22]. CANDLE $(n=11)$, SAVI $(n=7)$, NOMID $(n=18), \mathrm{HC}(n=26)$. JDM overall $(n=57)$ * $^{*} q<0.05,{ }^{* *} q<0.01$. JDM-HQ $(n=14):{ }^{* *} p<0.01$. JDM overall and JDM-HQ are higher than NOMID and HC; JDM overall is lower than CANDLE and SAVI.

Abbreviations: IRG, interferon-regulated gene; JDM, juvenile dermatomyositis; JDM-HQ, highest-quartile scores in JDM; CANDLE, Chronic Atypical Neutrophilic Dermatosis with Lipodystrophy and Elevated temperature; SAVI, STING-Associated Vasculopathy with onset during Infancy; NOMID, neonatal-onset multisystem inflammatory disease; $H C$, healthy controls; IQR, interquartile range

those of patients with CANDLE and SAVI. Anti-MDA5 autoantibody-positive and MSA-negative patients (median 147 and 186, respectively) are lower but not statistically different from CANDLE and SAVI (Additional file 1: Figure S1).

\section{Principal component analysis (PCA)}

All 5 PCA analyses had three interpretable principal components (PCs) with eigenvalues $>1$, accounting for $89.3-91.3 \%$ of the variance. All 5 PCAs produced a similar PC1 with high (>0.8) component loadings for 23-25 out of 28 genes accounting for $80.4-82.8 \%$ of overall variance and did not distinguish CANDLE, SAVI, and JDM well (Additional file 1: Table S3). Thus, PC1 differentiates IFN disease groups (CANDLE, SAVI, JDM, and JDM MSA groups) from IFN-negative controls (NOMID and $\mathrm{HC}$ ). PC2 and $\mathrm{PC} 3$ better demonstrated the 
differentiation among CANDLE, SAVI, and JDM overall (PCA-A, Fig. 2a), or the specific MSA groups within JDM from CANDLE and SAVI (PCA-B to PCA-D, Fig. 2b-d; PCA-E, Additional file 1: Figure S2). CANDLE, SAVI, and JDM or the specific MSA group separated from HC and NOMID in PC2 and PC3. JDM-HQ patients are further separated from $\mathrm{HC}$ and NOMID than the remaining JDM patients. In PC2 and PC3, JDM overlaps more with SAVI than CANDLE. Anti-MDA5 autoantibody-positive JDM patients show near-complete overlap with SAVI (PCA-B, Fig. 2b), in contrast to antiNXP2 and anti-TIF1 autoantibody-positive JDM, and the MSA-negative groups, which overlap more with SAVI than CANDLE, but to a lesser degree (PCA-C, PCA-D, Fig. 2c, d; PCA-E, Additional file 1: Figure S2).

Across all five PCAs, we identified the genes with the largest component loadings in PC2 and PC3 (absolute value $>0.4$ ). Briefly, component loadings are essentially weights representing the relative contributions of each gene to the principal component (linear combinations of individual gene scores). In 4 of the 5 PCAs (for JDM overall and all subgroups except anti-TIF1), PC2 had large loadings for GBP1 and SOCS1, and PC3 had large loadings for CXCL10 (Table 3). IFI27 was a large contributor to PC2 in PCAs that included JDM overall, as well as anti-TIF1 and anti-NXP2 autoantibody-positive patients, and a large PC3 contributor for PCAs that included anti-MDA5 autoantibody-positive and MSAnegative patients. LAMP3 had a large PC2 loading only for the PCA that included anti-MDA5 autoantibodypositive patients. The anti-TIF1 autoantibody-positive PCA had CXCL10 in PC2 and GBP1 and SOCS1 in PC3.

\section{Gene proportion analysis}

The contribution of individual genes to the normalized total IRG-S was evaluated among CANDLE, SAVI, and

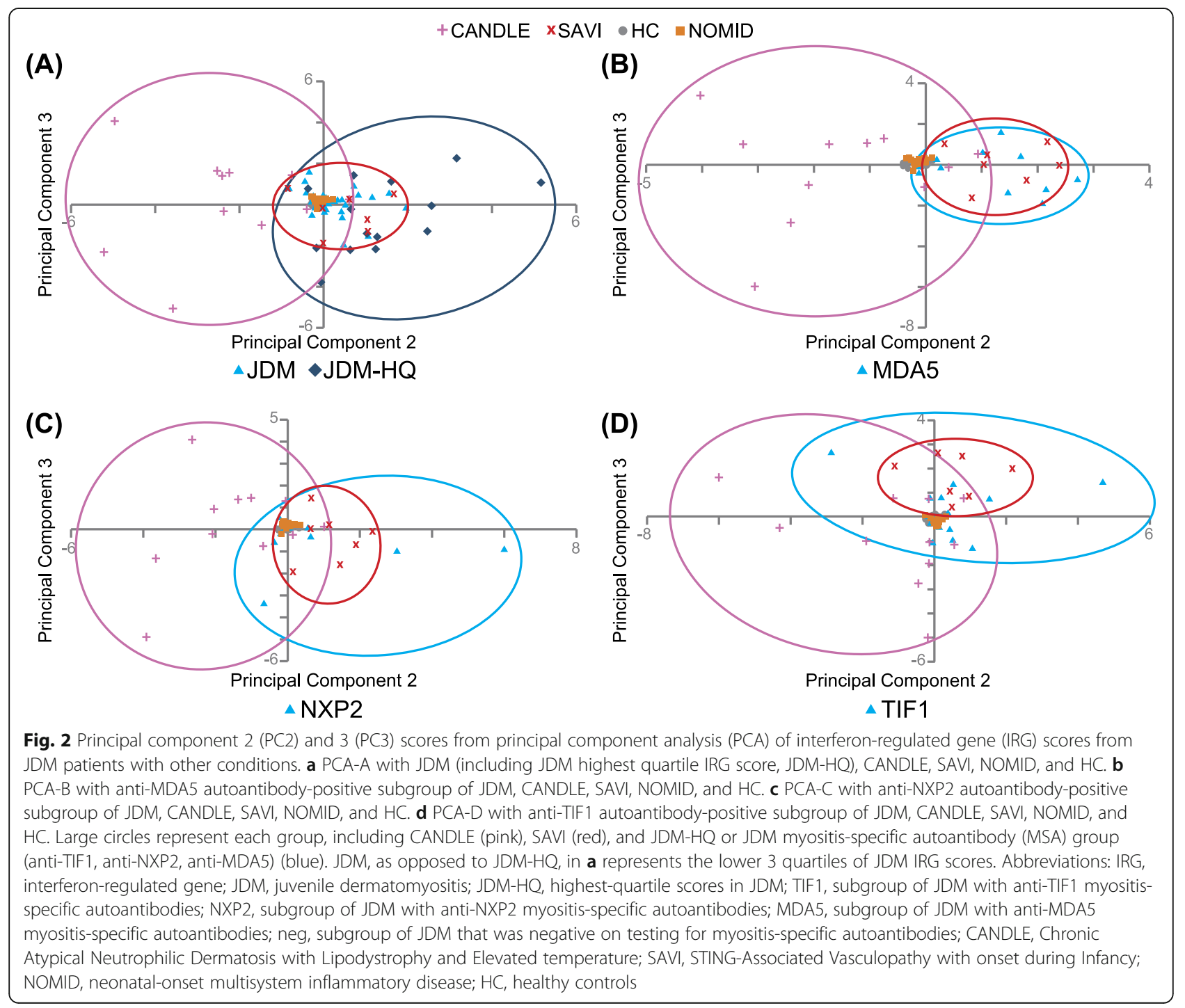


Table 3 Highest magnitude component loadings from PCA (PC2, PC3) for JDM/MSA subgroups versus other conditions

\begin{tabular}{|c|c|c|c|c|c|c|c|c|c|c|}
\hline \multirow[t]{2}{*}{ Gene } & \multicolumn{2}{|c|}{ PCA-A with JDM } & \multicolumn{2}{|c|}{ PCA-B with anti-MDA5 Ab } & \multicolumn{2}{|c|}{ PCA-C with anti-NXP2 Ab } & \multicolumn{2}{|c|}{ PCA-D with anti-TIF1 Ab } & \multicolumn{2}{|c|}{ PCA-E with MSA-neg } \\
\hline & PC2 & PC3 & PC2 & PC3 & PC2 & PC3 & PC2 & PC3 & PC2 & PC3 \\
\hline CXCL10 & -0.102 & -0.667 & -0.267 & -0.666 & -0.321 & -0.685 & -0.659 & $\underline{0.336}$ & -0.259 & -0.686 \\
\hline GBP1 & -0.506 & $\underline{0.215}$ & -0.562 & 0.167 & -0.448 & $\underline{0.216}$ & -0.200 & -0.473 & 0.517 & -0.076 \\
\hline$|F| 27$ & -0.510 & -0.345 & -0.218 & -0.467 & -0.444 & -0.250 & -0.579 & 0.062 & 0.145 & -0.546 \\
\hline LAMP3 & -0.216 & 0.059 & -0.444 & 0.049 & -0.325 & -0.021 & -0.066 & -0.311 & $\underline{0.349}$ & -0.177 \\
\hline SIGLECI & 0.132 & -0.424 & 0.338 & -0.330 & 0.017 & -0.348 & -0.204 & $\underline{0.315}$ & -0.343 & -0.224 \\
\hline SOCS1 & -0.428 & $\underline{0.300}$ & -0.437 & 0.155 & -0.413 & $\underline{0.323}$ & -0.085 & -0.503 & 0.486 & 0.061 \\
\hline
\end{tabular}

Bold indicates an absolute value of component loading $>0.4$ (high magnitude)

Italic and underline indicates an absolute value of component loading between 0.2 to 0.4

PCA-A: PCA of JDM, CANDLE, SAVI, NOMID, and HC

PCA-B: PCA of Anti-MDA5 Ab, CANDLE, SAVI, NOMID, and HC

PCA-C: PCA of Anti-NXP2 Ab, CANDLE, SAVI, NOMID, and HC

PCA-D: PCA of Anti-TIF1 Ab, CANDLE, SAVI, NOMID, and HC

PCA-E: PCA of MSA-neg, CANDLE, SAVI, NOMID, and HC

The component loadings are the correlations of the individual gene normalized Z-scores with the given principal component. A stronger loading (e.g., greater than 0.40 or less than -0.40 ) indicates a stronger relationship or contribution of that gene with that principal component. As with correlations, these can be negative or positive. The highest magnitude component loading interferon-regulated genes are listed on the left. The sign or direction of the component loading is arbitrary and can be reversed with the same interpretation, so the focus should be on the weight or magnitude

Abbreviations: PCA principal component analysis, PC principal component, JDM includes all juvenile dermatomyositis patients, MSA myositis-specific autoantibody group, anti-TIF1 Ab includes subgroup of JDM patients with anti-TIF1 autoantibodies, anti-NXP2 Ab subgroup of JDM patients with anti-NXP2 autoantibodies, antiMDA5 $A b$ includes subgroup of JDM patients with anti-MDA5 autoantibodies, MSA-neg includes subgroup of JDM that is negative for myositis-specific

autoantibodies, CANDLE Chronic Atypical Neutrophilic Dermatosis with Lipodystrophy and Elevated temperature, SAVI STING-Associated Vasculopathy with onset during Infancy, NOMID neonatal-onset multisystem inflammatory disease

JDM-HQ $(n=14)$. JDM-HQ was chosen for comparison as overall, it has IRG-S in the same range as CANDLE and SAVI in order to compare gene proportions more equitably. IFI27 constituted a significantly smaller proportion of the 28-gene IRG-S in the JDM-HQ (median $6.9 \%$ ) than in CANDLE (median $32.4 \%$ ) or SAVI (median 27.0\%) (Fig. 3a). Because IFI27 dominated the IRG$\mathrm{S}$ in CANDLE and SAVI and the proportion of total IRG-S was higher in JDM-HQ for many other IRGs, analysis comparing contribution of other genes to the overall score was done after removing IFI27. Without IFI27, only IFIT1 remained significantly greater in proportion in JDM-HQ versus SAVI and CANDLE. GBP1 continued to constitute a greater percentage of the IRG$\mathrm{S}$ in CANDLE than JDM-HQ, while four IRGs (HERC5, $M X 1, O A S 3, O A S L)$ had significantly greater proportion in JDM-HQ than in CANDLE (Fig. 3b).

\section{Ratios related to NF-KB and IFN- $\gamma$}

The NF- $k B$ ratio in the JDM patients with elevated IRG$\mathrm{S}$ (median 0.03) was significantly lower than NOMID (median 0.13) and HC (median 0.10). They were in the range of the CANDLE (median 0.06) and SAVI (median 0.02 ) patients, consistent with a smaller cohort of JDM patients previously assessed (Additional file 1: Figure S3A) [26]. When examining JDM patients by the MSA group, the anti-MDA5 group (median 0.02 ) has the lowest ratio (Additional file 1: Figure S3B). Regarding the IFN- $\gamma$ ratio, the JDM patients with elevated IRG-S have a significantly higher ratio (median 0.44 ) versus CANDLE (median 0.38) and also have a higher ratio than SAVI (median $0.41, p=0.06$ ) (Additional file 1: Figure S4A). When examining JDM patients by MSA groups, the anti-MDA5 group (median 0.44 ) has the lowest ratio (Additional file 1: Figure S4B).

\section{IRG score correlations in JDM}

The standard IRG-S correlated moderately $\left(r_{s}=0.33-\right.$ 0.47 ) with seven measures of disease activity in JDM, including physician global activity (PGA), manual muscle testing (MMT), extra-muscular global and skeletal activity, Disease Activity Score (DAS), and 2 serum muscle enzyme levels, with similar performance 2 published IRG-S (Table 2). In anti-TIF1 autoantibody-positive JDM patients, IRG-S more strongly correlated with cutaneous and extra-muscular activity, as well as DAS total and skin activity $\left(r_{s}=0.58-0.76\right)$, with lower correlation with MMT. Other IRG-S performed similarly for the anti-TIF1 autoantibody-positive JDM group (data not shown). Limited patient assessments precluded correlation of IRG-S with disease measures in anti-NXP2 and anti-MDA5 autoantibody-positive patients, although there was no difference in PGA among MSA subgroups.

Forty-six percent $(26 / 57)$ of JDM patients had high IRG-S (> 48.9). JDM patients with high IRG-S (> 48.9) differed in 11 disease activity measures from those with low scores (Additional file 1: Table S1). From the multivariable regression analysis, low muscle strength (odds ratio $[\mathrm{OR}] 0.91, p<0.01$ ) and high skeletal (joint) disease activity (OR 3.3, $p=0.03$ ) were the best predictors of high IRG-S (Table 4). No significant differences in high versus low IRG-S were found by gender, race, disease 


\begin{tabular}{|c|c|c|c|}
\hline \multicolumn{4}{|l|}{ (A) } \\
\hline Gene & JDM-HQ & CANDLE & S AVI \\
\hline CXCL10 & $1.7[0.7-4.4]$ & $0.5[0.3-2.2]$ & $0.4[0.1-1.6]$ \\
\hline DDX60 & $3.2[2.8-4.1]$ & $2.8[2.3-4.2]$ & $3.0[2.5-3.7]$ \\
\hline EPSTII* & $4.4[3.8-5.5]$ & $3.7[2.6-4.2]$ & $3.3[2.7-3.6]$ \\
\hline GBP1† & $1.4[0.9-2.1]$ & $3.4[1.7-4.9]$ & $0.9[0.5-1.1]$ \\
\hline HERC5: & $2.5[1.8-3.7]$ & $1.2[0.6-1.9]$ & $1.7[1.2-2.0]$ \\
\hline HERC6 & $3.3[3.0-4.1]$ & $2.2[1.8-3.0]$ & $3.1[3.0-4.1]$ \\
\hline IFI27\% & $6.9[2.3-21.1]$ & $32.4[15.9-46.3]$ & $27.0[21.2-43.5]$ \\
\hline IFI44 & $4.4[3.7-5.1]$ & $3.5[2.5-4.7]$ & $3.4[3.1-4.0]$ \\
\hline IFI44L & $5.6[5.1-6.1]$ & $4.9[3.6-5.7]$ & $5.1[4.9-6.5]$ \\
\hline IFI6 & 2.5 [1.9-3.1] & $1.8[1.2-2.5]$ & $1.5[1.1-2.3]$ \\
\hline IFIT1f & $2.9[2.6-3.7]$ & $1.4[0.7-1.9]$ & $1.9[1.2-2.1]$ \\
\hline IFIT2 & 2.5 [1.4-4.3] & $1.7[0.3-1.9]$ & $1.7[1.2-2.3]$ \\
\hline IFIT3\% & $2.9[2.1-3.5]$ & $1.9[1.3-3.0]$ & $2.0[1.3-2.1]$ \\
\hline IFIT5 & $2.7[2.3-4.3]$ & $2.2[1.4-2.5]$ & $2.7[1.7-2.8]$ \\
\hline ISG15 & $3.5[3.4-4.2]$ & $3.3[2.3-4.2]$ & $3.8[3.1-4.6]$ \\
\hline$L A M P 3$ & $0.8[0.6-0.9]$ & $0.6[0.4-1.1]$ & $0.2[0.1-0.4]$ \\
\hline$L Y 6 E$ & $3.7[3.3-5.8]$ & $3.7[2.7-5.1]$ & $4.3[4.0-4.9]$ \\
\hline$M X 1+$ & $2.3[1.9-3.5]$ & $\underline{1.2[0.7-1.6]}$ & $1.2[1.1-2.3]$ \\
\hline$O A S 1$ & $3.0[2.3-4.0]$ & $3.0[2.4-3.7]$ & $2.9[2.6-3.7]$ \\
\hline$O A S 2 \dagger$ & $3.8[3.3-4.9]$ & $2.4[1.7-2.9]$ & $3.1[2.6-3.8]$ \\
\hline$O A S 3 \neq$ & $3.4[2.8-4.1]$ & $1.8[1.1-2.5]$ & $2.2[1.8-2.6]$ \\
\hline$O A S L \dagger$ & $2.2[1.8-2.9]$ & $\underline{1.3[0.6-1.8]}$ & $2.2[1.4-2.6]$ \\
\hline$R S A D 2$ & $3.1[2.6-4.5]$ & $2.7[1.6-3.4]$ & $2.6[2.4-3.3]$ \\
\hline RTP4 & $3.8[3.1-4.6]$ & $4.7[2.7-5.0]$ & $4.0[3.4-5.2]$ \\
\hline SIGLEC1 & $2.9[2.0-4.3]$ & $1.8[1.3-2.0]$ & $2.1[0.9-4.4]$ \\
\hline SOCS1 & $1.7[0.6-2.8]$ & $2.3[0.9-3.7]$ & $0.8[0.7-1.0]$ \\
\hline SPATS2L & $2.8[1.7-3.5]$ & $2.7[2.3-3.2]$ & $2.0[1.3-2.7]$ \\
\hline USP18† & $5.0[4.2-6.1]$ & $\underline{3.7[2.0-4.4]}$ & $3.6[3.2-5.1]$ \\
\hline
\end{tabular}

(B)

\begin{tabular}{|c|c|c|c|}
\hline Gene & JDM-HQ & CANDLE & S AVI \\
\hline CXCL10 & $1.9[0.8-4.7]$ & $0.9[0.5-2.3]$ & $0.7[0.1-2.1]$ \\
\hline DDX60 & $3.8[3.2-4.3]$ & $4.2[3.7-4.6]$ & $4.5[3.8-5.1]$ \\
\hline EPSTII & $4.5[4.4-6.0]$ & $5.4[4.8-5.9]$ & $4.5[4.5-5.0]$ \\
\hline GBP1 & $1.4[1.1-2.7]$ & $5.6[3.3-6.0]$ & $1.2[0.7-1.5]$ \\
\hline HERC5† & $2.6[2.4-3.8]$ & $1.8[1.5-2.1]$ & $2.5[1.6-2.6]$ \\
\hline HERC6 & $4.0[3.0-5.3]$ & $3.6[2.7-4.8]$ & $5.2[4.6-5.7]$ \\
\hline IFI44 & $5.2[4.7-5.3]$ & $5.2[4.7-5.4]$ & $5.0[4.6-5.7]$ \\
\hline IFI44L & $6.4[5.7-7.0]$ & $6.7[6.5-8.0]$ & $7.7[7.0-9.7]$ \\
\hline IFI6 & $2.9[2.4-3.3]$ & $2.6[2.2-3.4]$ & $2.2[1.9-3.5]$ \\
\hline IFIT1宗 & $3.5[2.9-4.1]$ & $2.0[1.7-2.6]$ & $2.5[1.8-3.0]$ \\
\hline IFIT2 & $2.7[1.5-4.9]$ & $2.0[0.7-2.8]$ & $2.4[1.5-3.1]$ \\
\hline IFIT3 & $3.4[2.8-3.8]$ & $2.8[2.5-4.2]$ & $2.6[2.2-3.2]$ \\
\hline IFIT5 & $3.1[2.7-4.3]$ & $3.0[2.6-3.7]$ & $3.1[3.0-3.8]$ \\
\hline ISG15 & $4.3[3.5-5.0]$ & $4.9[3.8-6.5]$ & 5.4 [4.4-6.9] \\
\hline LAMP3 & $0.9[0.7-1.1]$ & $1.1[0.6-1.5]$ & $0.3[0.3-0.5]$ \\
\hline LY6E & $4.5[4.1-6.1]$ & $5.3[4.7-6.6]$ & $5.6[5.6-7.1]$ \\
\hline$M X 1+$ & $2.6[2.3-3.5]$ & $\underline{1.7[1.7-2.1]}$ & $2.1[1.9-2.8]$ \\
\hline OAS1 & $3.3[2.8-4.1]$ & $4.3[3.5-5.1]$ & $4.5[4.2-4.8]$ \\
\hline$O A S 2$ & $4.2[4.0-5.0]$ & $3.3[2.8-4.1]$ & $4.8[4.0-5.6]$ \\
\hline$O A S 3 \dagger$ & $3.7[3.4-4.3]$ & $\underline{2.8[2.1-3.0]}$ & $3.3[3.0-3.4]$ \\
\hline OASL' & $2.6[1.9-3.1]$ & $1.8[1.6-2.2]$ & $2.9[2.5-3.3]$ \\
\hline$R S A D 2$ & $3.6[2.9-4.6]$ & $3.8[3.2-4.3]$ & $3.7[3.4-4.5]$ \\
\hline RTP4 & $4.4[3.3-5.5]$ & $6.1[5.0-7.4]$ & $5.7[5.2-6.9]$ \\
\hline SIGLEC1 & $3.5[2.1-5.6]$ & $2.3[2.1-3.5]$ & $2.4[1.3-6.1]$ \\
\hline SOCS1 & $2.0[0.8-3.0]$ & $3.0[2.2-4.5]$ & $1.1[0.9-1.9]$ \\
\hline$S P A T S 2 L$ & $3.0[2.1-4.2]$ & $4.1[3.7-5.2]$ & $2.8[2.1-3.5]$ \\
\hline USP18 & $5.8[4.6-7.6]$ & $4.8[2.9-5.6]$ & $5.9[4.9-6.7]$ \\
\hline
\end{tabular}

Fig. 3 Proportion of individual genes from 28 normalized IRG-S for JDM highest quartile (JDM-HQ), CANDLE, SAVI. *JDM-HQ gene proportion significantly different from SAVI. ${ }^{\dagger} J D M-H Q$ gene proportion significantly different from CANDLE. ${ }^{\ddagger} J D M-H Q$ gene proportion significantly different from CANDLE and SAVI. Heat map of median proportion of the respective gene by normalized Z-score with red for highest proportion and dark blue for lowest proportion. Bold indicates values in a diagnosis group(s) significantly higher compared to values in the italicized/underlined group(s). Italicized/underlined indicates values in a diagnosis group(s) significantly lower compared to values in bold group(s). a All 28 IRGs are included. b IFI27 has been removed. Individual gene percentages (\%) of the total IRG score, by normalized Z-scores (see the "Methods" section) are shown in alphabetical order by gene as median and interquartile ranges for JDM highest-quartile, CANDLE, and SAVI patients. Medians percentages of each gene by diagnosis may not sum to 100 due to skewing in the group, but they do in individual patients. JDM-HQ $(n=14)$ included 4 with anti-TIF1 Ab, 4 with anti-MDA4 Ab, 3 with anti-NXP2 Ab, and 3 MSA-neg. The JDM Physician Global Activity (PGA) median is 4.1/ 10 with an interquartile range of 2.4 to 4.7. Abbreviations: IRG, interferon-regulated gene; JDM-HQ, juvenile dermatomyositis patients with the highest quartile of IRG scores; CANDLE, Chronic Atypical Neutrophilic Dermatosis with Lipodystrophy and Elevated temperature; SAVI, STING associated Vasculopathy with onset during Infancy; anti-TIF1 Ab, includes subgroup of JDM patients with anti-TIF1 autoantibodies; anti-NXP2 Ab, subgroup of JDM patients with anti-NXP2 autoantibodies; anti-MDA5 Ab, includes subgroup of JDM patients with anti-MDA5 autoantibodies; MSA-neg, includes subgroup of JDM that is negative for myositis-specific autoantibodies; PGA. Physician Global Activity on 0-10 visual analog scale

duration, or ever having calcinosis, cutaneous ulceration, or interstitial lung disease (Additional file 1: Table S1). Unfortunately, small numbers within specific MSA groups precluded multivariable regression analysis within a MSA group.
Regarding therapy (Additional file 1: Table S4), neither oral nor intravenous corticosteroid doses correlated with IRG-S $\left(r_{s}=0.04-0.08\right)$. No differences in IRG-S were evident among patients grouped by total steroid-sparing medications received. Patients receiving hydroxychloroquine 
Table 4 Multivariable logistic regression predicting high IFN score in JDM patients

\begin{tabular}{llllllc}
\hline Parameter & Estimate & Standard error & $p$ value & Odds ratio & \multicolumn{2}{c}{ 95\% confidence interval } \\
\cline { 5 - 7 } & & & & & Lower \\
\hline Constant & 23.247 & 8.968 & $\mathbf{0 . 0 1 0}$ & - & - & - \\
Total MMT score & -0.099 & 0.037 & $\mathbf{0 . 0 0 7}$ & 0.91 & 0.84 & 0.97 \\
LDH & -0.013 & 0.007 & 0.073 & 0.99 & 0.97 & 1.00 \\
Skeletal VAS & 1.184 & 0.550 & $\mathbf{0 . 0 3 1}$ & 3.27 & 1.11 & 9.60 \\
\hline
\end{tabular}

High and low 28 interferon-regulated gene (IRG) score is defined by above and below 48.9, 95th percentile of healthy controls [22]. Backwards stepwise regression was performed (alpha < 0.15). $p$ values $<0.05$ are bolded. With the odds ratio, 1 is 1 point in the MMT score, LDH value, or 1 point on 10 point scale of skeletal VAS

Abbreviations: Total MMT score 26 muscle manual muscle testing (0-260), LDH lactate dehydrogenase, skeletal VAS Myositis Disease Activity Assessment Tool Skeletal disease activity visual analog scale

( $n=21 / 56)$, methotrexate $(n=40 / 56)$, or intravenous immunoglobulin $(n=18 / 56)$ had no difference in IRG-S compared to those not receiving these therapies. Patients receiving mycophenolate mofetil (MMF) $(n=8 / 56)$ had lower IRG-S than those not receiving MMF (median - 4.29 vs. 85.2, $p=0.004)$, although they also had significantly lower PGA (median 1.7 vs. 2.3, $p=0.03$ ).

Given the increased overlap of the anti-MDA5 subgroup of the JDM cohort ( $n=10$ with clinical information), we assessed the clinical characteristics of this group in particular versus the rest of JDM, CANDLE, and SAVI (Additional file 1: Table S2). The anti-MDA5 subgroup of JDM versus the rest of JDM was characterized by increased cutaneous ulceration $(7 / 10,70 \%$ vs. $8 /$ 46, 17\%) and interstitial lung disease (ILD) (7/10, 70\% vs. $1 / 46,2 \%)$ with less weakness defined as a MMT score of $<225 / 260(0 / 8,0.0 \%$ vs. $8 / 28,29 \%)$. In our SAVI cohort, distal ulcerations $(4 / 4,100.0 \%)$ and ILD (5/5, $100.0 \%$ ) are common, while both are not features commonly observed in our CANDLE cohort (none reported and $3 / 9,33.3 \%$, respectively). Myositis is not a frequently observed or assessed feature in our SAVI patients $(1 / 4$, 25.0\%) and more common in our CANDLE cohort (8/ $10,80.0 \%)$ though MMT was not performed. Overall, there are multiple features overlapping between JDM, CANDLE, and SAVI with the highest overlap between anti-MDA5 autoantibody-positive JDM patients with SAVI.

To compare the interferon-regulated gene expression to protein expression, the IRG-S was compared to IP-10 in JDM $(n=19)$. A significant correlation was found $\left(r_{s}=\right.$ 0.49, $p=0.03$ ), similar to that observed in CANDLE and SAVI patients [20].

\section{Discussion}

This study analyzed an IRG-S in JDM and MSA groups within JDM versus Mendelian interferonopathies (CANDLE and SAVI), an IL-1 mediated, non-IFN autoinflammatory disease control (NOMID), and healthy controls. PCA and gene proportion analysis suggested similarities of the IRG pattern elevation in JDM and anti-MDA5 autoantibody-positive patients to SAVI and a moderate correlation of IRG-S with clinical measures.

Blood IRG-S in JDM patients ranged from the levels in $\mathrm{HC}$ to as high as CANDLE, and SAVI, but were primarily between HC/NOMID and CANDLE/SAVI. We confirmed that some JDM patients' IRG-S overlap with HC (54\% in our cohort) [7, 28]. IFI27 was found to be most dynamically upregulated [22], contributing a higher proportion when the IRG-S is higher (data not shown). We found that although IFI27 contributed the most to the total IRG-S in JDM-HQ, SAVI, and CANDLE with similarly high overall scores, the proportion of IFI27 to the total IRG-S was lower in JDM-HQ. After excluding IFI27, four genes (HERC5, MX1, OAS3, OASL) contributed a higher proportion to the total IRG-S in JDM-HQ versus CANDLE, whereas one gene, IFIT1, had an increased proportion in JDM-HQ compared to both CANDLE and SAVI. IFIT1 is a negative regulator of STING, which is an adaptor protein essential for IFN- $\beta$ induction. IFIT1 disrupts the STING interaction with mitochondrial anti-viral signaling protein (MAVS) and TANK-binding kinase (TBK1), which decreases IFN- $\beta$ expression [29], and may present a counter-regulatory mechanism to "dampen" STING and IFN- $\beta$ signaling in JDM. Interestingly, SAVI, caused by gain-of-function mutations in STING leading to constitutive activation of IFN- $\beta$ through the STING-TBK1-IRF3 pathway [16], has a more complete overlap of IRG-S with JDM than CANDLE suggesting that STING and IFN- $\beta$ may be preferentially activated in JDM. In DM, the higher correlation of serum IFN- $\beta$ with disease activity and peripheral blood IRG signature versus IFN- $\alpha$ [10] could be consistent with a pathogenic role of STING in JDM. JDM patients with elevated IRG-S had lower NF- $\kappa B$ ratios, similar to canonical interferonopathies CANDLE and SAVI, indicating it is unlikely JDM has much concomitant NF-kB signaling seen in other conditions with an elevated IRG$S$ [26]. Overall, JDM with elevated IRG-S had higher IFN- $\gamma$ ratios than CANDLE and SAVI, which is consistent with previous reports of IFN- $\gamma$ co-localizes with 
$\mathrm{CD}^{+} \mathrm{T}$ cells in untreated JDM muscle biopsies and increase in both type I and type I interferon-regulated genes in JDM muscle [8], which may differentiate JDM from autoinflammatory CANDLE and SAVI. Differences in these ratios by MSA groups merit evaluation in larger groups.

SAVI is a vasculopathy characterized by violaceous plaques and/or nodules, often with features of vascular damage with gangrene and infarcts in acral areas such as at the tips of the fingers and/or toes [16, 17]. JDM is also a small-vessel vasculopathy with dilated and tortuous periungual capillaries, endarteropathy, and capillary loss noted on muscle biopsy, and patients may develop vasomotor instability in acral areas [3, 30,31]. Both JDM and SAVI share features of vasculopathy leading to thrombosis, tissue ischemia, and infarction, consistent with both phenotypic overlap and potentially shared role of IFN in vasculopathy pathogenesis $[16,17,30,31]$. Although Janus kinase (JAK) inhibitor therapy does not specifically or fully block IFN- $\beta$ or constitutively activated STING, it does inhibit type I and II IFN signaling [32] and several reports have noted clinical improvement in SAVI with decreased skin vasculopathy and stabilization of lung disease on JAK inhibitor therapy [20, 33]. Similarly, a limited number of cases in refractory JDM patients have noted improvement of skin and muscle symptoms with JAK inhibitor therapy [34, 35, 38].

MSA groups continue to evolve as an important predictor of subgroups within JDM both regarding phenotype and prognosis. Among the IRG-S of MSA group patients, the anti-MDA5 autoantibody-positive IRG-S overlapped most closely with SAVI by PCA. The antiMDA5 autoantibody subgroup of JDM is characterized by increased cutaneous ulceration and interstitial lung disease (ILD) with less muscle disease [1-3], also observed in our cohort, with more overlap with these vasculopathic features seen in SAVI than CANDLE in general [15-17], also specifically observed in our cohort. JAK inhibitor therapy has been noted to show improved outcomes in refractory anti-MDA5 autoantibody adult and juvenile dermatomyositis, including with rapidly progressive ILD [36-38].

Furthermore, the peripheral blood IRG-S correlates moderately with disease activity measures, including muscle and extra-muscular components $[6,7,10]$. Multivariable regression analysis identified weakness (MMT-26) and increased skeletal features (skeletal VAS) as the most important factors associated with high IRG$\mathrm{S}$ in JDM overall. MSA-negative patients having elevated IRG-S differs from previous reports of lower IFN chemokine scores in MSA-negative patients, although this was a different type of score and the methodology to determine MSAs also differed [39]. In anti-TIF1 autoantibody-positive patients, who characteristically tend to have more photosensitive rashes, the IRG-S correlated more strongly with skin disease-activity measures although anti-TIF1 patients did not have significantly higher skin activity in our cohort. Unfortunately, small numbers of patients precluded multivariable regression analysis within the anti-TIF1 MSA group.

Though type I IFN itself is in extremely low concentrations in the blood or serum, IRGs are readily quantifiable and have been associated with elevation of serum IFN- $\alpha$ and IFN- $\beta$ in DM $[7,10]$. Notably, our gene expression assessment on NanoString ${ }^{\text {twx }}$, which does not require amplification, has low inter-assay and inter-observer variability and excellent reproducibility [22] and may hold value as a potential biomarker in the clinical setting compared to a traditional polymerase chain reaction or PCR-based methods used in other studies $[6,7,28]$ that are more difficult to standardize particularly across centers. Additionally, the IRG-S correlated significantly with peripheral protein IP-10. The "gold standard" to validate the peripheral IFN score would be a comparison to the affected skin and/or muscle, in which elevated IRG expression has been previously reported [8, 9]. Although correlations between skin/muscle and blood IRG expression have not been performed, the correlation of the blood IRG expression with muscle and skin disease activity in our Nanostring $^{\text {Tw }}$ assay and other reports using PCR analyses $[6,7,10]$ holds promise as a valuable disease activity marker, though further validation is needed.

Limitations of this study include the evaluation of referred JDM patients at a single time point who have received multiple immunosuppressive therapies. We generally did not find that the IRG-S correlated with any specific medications. However, muscle IRG expression in DM and adult polymyositis and peripheral interferonregulated chemokine score in refractory adult and pediatric myositis was reported to be reduced with rituximab therapy [40, 41]. The number of patients with Mendelian autoinflammatory diseases and within MSA subgroups was small, but the uniform elevation of the IRG signature and disease-specific differences still allowed for meaningful analysis. Lastly, we evaluated whole blood; thus, the differential contributions of various cell types to the IRG elevation could not be assessed. Though we could not normalize for specific different cell populations within whole blood, it is reassuring that exploratory analysis of white blood cells and differential cell counts between JDM versus CANDLE and SAVI did not find any significant differences (data not shown).

\section{Conclusions}

In conclusion, the IRG-S correlates with disease measures in JDM, with a higher correlation for cutaneous features in the anti-TIF1 autoantibody group. IRG-S in JDM has similarities to that of Mendelian 
interferonopathies, particularly IRG-S for the JDM-HQ and anti-MDA5 autoantibody subgroups. Further evaluation by the MSA group in larger cohorts is important to examine. Specific IRGs have a differential contribution in JDM patients, with a pattern more similar to SAVI than CANDLE, pointing to a potentially larger role of STING activation and IFN- $\beta$ in JDM and a potential treatment target.

\section{Supplementary information}

Supplementary information accompanies this paper at https://doi.org/10. 1186/s13075-020-02160-9.

Additional file 1: Table S1. Univariate Comparison of Parameters in JDM patients with High versus Low Interferon-Regulated Gene Score. Table S2. Clinical Features of JDM and myositis-specific autoantibody (MSA) groups, CANDLE, and SAVI. Table S3. Component loadings for Principal Component (PC) Analysis for PC1, PC2 and PC3 for JDM or myositis-specific autoantibody (MSA) subgroups of JDM, with autoinflammatory conditions and controls. Table S4. Treatment information. Figure S1. Peripheral blood interferon-regulated gene (IRG) scores in juvenile dermatomyositis (JDM) myositis-specific autoantibody (MSA) groups compared to monogenic autoinflammatory disease patients and healthy controls. Figure S2. PCA graph of Principal Component 2 and 3 scores with MSA-negative JDM group with other conditions. Figure S3. NF-KB ratio in JDM with elevated IRG-S and by MSA groups versus other conditions. Figure S4. IFNY ratio in JDM with elevated IRG-S and by MSA groups versus other conditions.

\section{Abbreviations}

JDM: Juvenile dermatomyositis; IFN: Interferon; CANDLE: Chronic Atypical Neutrophilic Dermatosis with Lipodystrophy and Elevated temperature: SAVI: STING-Associated Vasculopathy with onset in Infancy; IRG-S: Interferonregulated gene score; MSA: Myositis-specific autoantibodies; HC: Healthy controls; PCA: Principal component analysis; IRG: Interferon-regulated genes; DM: Dermatomyositis; STING: STimulator of INterferon Genes; JAK: Janus kinase; NOMID: Neonatal-onset multisystem inflammatory disease; $\mathrm{NIH}$ : National Institutes of Health; NIEHS: National Institute of Environmental Health Sciences; IRB: Institutional Review Board; NIAID: National Institute of Allergy and Infectious Diseases; JDM-HQ: Highest quartile of juvenile dermatomyositis interferon-regulated gene scores; MDAAT: Myositis Disease Activity Assessment Tool; VAS: Visual analog scale; PC: Principal component; PGA: Physician global activity assessment by visual analog scale; MMT: Manual muscle testing; DAS: Disease Activity Score; MMF: Mycophenolate mofetil; ILD: Interstitial lung disease; MAVS: Mitochondrial anti-viral-signaling protein; TBK1: TANK-binding kinase; PCR: Polymerase chain reaction; IP-10: Interferon gamma-inducible protein 10; NF-kB: Nuclear factor kappa B

\section{Acknowledgements}

The authors thank Drs. Sarthak Gupta and Robert Colbert for their critical reading of the manuscript.

\section{Authors' contributions}

Study concept and design was by HK, RGM, and LR. HK, FGR, JM, MG, EL, LR, and RGM analyzed and interpreted the data. HK and WT performed the experiments. $I T, Y H, T O$, and ADJ acquired the data. LR, FM, and RGM provided clinical samples and clinical data. HK, JM, ADJ, LR, and RGM made critical revisions to the paper. The the authors have read and approved of the final manuscript.

\section{Authors' information}

Raphaela Goldbach-Mansky and Lisa G. Rider share last authorship.

\section{Funding}

This work was supported by the Intramural Research Program of the National Institutes of Health, National Institute of Arthritis and Musculoskeletal and Skin Diseases including the Office of Science and
Technology and the Translational Immunology Section, National Institute of Environmental Health Sciences, National Institute of Allergy and Infectious Diseases, and NIH Clinical Center. Dr. Kim's work was supported in part by the Cure JM Foundation. Social \& Scientific Systems was supported under a contract with NIEHS (HHSN273201600002I).

\section{Availability of data and materials}

The datasets generated and analyzed during the current study are not publicly available due to their containing information that could compromise research participant privacy. The data are available and de-identified from the corresponding author (HK) on reasonable request.

\section{Ethics approval and consent to participate}

This study was carried out in accordance with the institutional review board (IRB) of the National Institutes of Health. All JDM patients consented to a NIEHS IRB-approved protocol. All CANDLE, SAVI, NOMID, and HC patients consented to a NIAID IRB-approved protocol (NCT02974595).

\section{Consent for publication}

Not applicable.

\section{Competing interests}

The authors declare that they have no competing interests.

\section{Author details}

${ }^{1}$ Pediatric Translational Research Branch, National Institute of Arthritis and Musculoskeletal and Skin Diseases, NIH, Bethesda, MD, USA. ${ }^{2}$ Environmental Autoimmunity Group, Clinical Research Branch, National Institute of Environmental Health Sciences, NIH, Bethesda, MD, USA. ${ }^{3}$ Social \& Scientific Systems, Inc., Durham, NC, USA. ${ }^{4}$ Translational Autoinflammatory Diseases Section, National Institute of Allergy and Infectious Diseases, NIH, Bethesda, MD, USA. ${ }^{5}$ VA Medical Center, University of Oklahoma Health Sciences Center, and Oklahoma Medical Research Foundation, Oklahoma City, OK, USA. ${ }^{6}$ Translational Immunology Section, National Institute of Arthritis and Musculoskeletal and Skin Diseases, NIH, Bethesda, MD, USA.

Received: 11 November 2019 Accepted: 23 March 2020

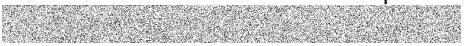

\section{References}

1. Pachman LM, Khojah AM. Advances in juvenile dermatomyositis: myositis specific antibodies aid in understanding disease heterogeneity. J Pediatr. 2018;195:16-27.

2. Tansley SL, Simou S, Shaddick G, Betteridge ZE, Almeida B, Gunawardena H, Thomson W, Beresford MW, Midgley A, Muntoni F, et al. Autoantibodies in juvenile-onset myositis: their diagnostic value and associated clinical phenotype in a large UK cohort. J Autoimmun. 2017;84:55-64.

3. Rider LG, Nistala K. The juvenile idiopathic inflammatory myopathies: pathogenesis, clinical and autoantibody phenotypes, and outcomes. J Intern Med. 2016;280(1):24-38.

4. Habers GE, Huber AM, Mamyrova G, Targoff IN, O'Hanlon TP, Adams S, Pandey JP, Boonacker C, van Brussel M, Miller FW, et al. Brief report: association of myositis autoantibodies, clinical features, and environmental exposures at illness onset with disease course in juvenile myositis. Arthritis Rheumatol. 2016:68(3):761-8.

5. Miller FW, Lamb JA, Schmidt J, Nagaraju K. Risk factors and disease mechanisms in myositis. Nat Rev Rheumatol. 2018;14(5):255-68.

6. Bilgic H, Ytterberg SR, Amin S, McNallan KT, Wilson JC, Koeuth T, Ellingson S, Newman B, Bauer JW, Peterson EJ, et al. Interleukin-6 and type I interferonregulated genes and chemokines mark disease activity in dermatomyositis. Arthritis Rheum. 2009;60(11):3436-46.

7. Greenberg SA, Higgs BW, Morehouse C, Walsh RJ, Kong SW, Brohawn P, Zhu W, Amato A, Salajegheh M, White B, et al. Relationship between disease activity and type 1 interferon- and other cytokine-inducible gene expression in blood in dermatomyositis and polymyositis. Genes Immun. 2012:13(3):207-13

8. Moneta GM, Pires Marafon D, Marasco E, Rosina S, Verardo M, Fiorillo C, Minetti C, Bracci-Laudiero L, Ravelli A, De Benedetti F, et al. Muscle expression of type I and type II interferons is increased in juvenile dermatomyositis and related to clinical and histological features. Arthritis Rheumatol. 2019;71(6):1011-21. 
9. Wong D, Kea B, Pesich R, Higgs BW, Zhu W, Brown P, Yao Y, Fiorentino D. Interferon and biologic signatures in dermatomyositis skin: specificity and heterogeneity across diseases. PLoS One. 2012;7(1):e29161.

10. Huard C, Gulla SV, Bennett DV, Coyle AJ, Vleugels RA, Greenberg SA. Correlation of cutaneous disease activity with type 1 interferon gene signature and interferon beta in dermatomyositis. Br J Dermatol. 2017; 176(5):1224-30.

11. Baechler EC, Bauer JW, Slattery CA, Ortmann WA, Espe KJ, Novitzke J, Ytterberg SR, Gregersen PK, Behrens TW, Reed AM. An interferon signature in the peripheral blood of dermatomyositis patients is associated with disease activity. Mol Med. 2007;13(1-2):59-68.

12. Greenberg SA, Pinkus JL, Pinkus GS, Burleson T, Sanoudou D, Tawil R, Barohn RJ, Saperstein DS, Briemberg HR, Ericsson M, et al. Interferon-alpha/ beta-mediated innate immune mechanisms in dermatomyositis. Ann Neurol. 2005:57(5):664-78.

13. Fall N, Bove KE, Stringer K, Lovell DJ, Brunner HI, Weiss J, Higgins GC, Bowyer SL, Graham TB, Thornton S, et al. Association between lack of angiogenic response in muscle tissue and high expression of angiostatic ELR-negative CXC chemokines in patients with juvenile dermatomyositis: possible link to vasculopathy. Arthritis Rheum. 2005;52(10):3175-80.

14. Brehm A, Liu Y, Sheikh A, Marrero B, Omoyinmi E, Zhou Q, Montealegre G, Biancotto A, Reinhardt A, Almeida de Jesus A, et al. Additive loss-of-function proteasome subunit mutations in CANDLE/PRAAS patients promote type I IFN production. J Clin Invest. 2015;125(11):4196-211.

15. Liu Y, Ramot Y, Torrelo A, Paller AS, Si N, Babay S, Kim PW, Sheikh A, Lee CC, Chen $Y$, et al. Mutations in proteasome subunit beta type 8 cause chronic atypical neutrophilic dermatosis with lipodystrophy and elevated temperature with evidence of genetic and phenotypic heterogeneity. Arthritis Rheum. 2012;64(3):895-907.

16. Kim H, Sanchez GA, Goldbach-Mansky R. Insights from Mendelian interferonopathies: comparison of CANDLE, SAVI with AGS, monogenic lupus. J Mol Med (Berl). 2016;94(10):1111-27.

17. Liu Y, Jesus AA, Marrero B, Yang D, Ramsey SE, Montealegre Sanchez GA, Tenbrock K, Wittkowski H, Jones OY, Kuehn HS, et al. Activated STING in a vascular and pulmonary syndrome. N Engl J Med. 2014;371(6):507-18.

18. Rodero MP, Crow YJ. Type I interferon-mediated monogenic autoinflammation: the type I interferonopathies, a conceptual overview. J Exp Med. 2016;213(12):2527-38.

19. de Jesus AA, Canna SW, Liu Y, Goldbach-Mansky R. Molecular mechanisms in genetically defined autoinflammatory diseases: disorders of amplified danger signaling. Annu Rev Immunol. 2015;33:823-74.

20. Sanchez GAM, Reinhardt A, Ramsey S, Wittkowski H, Hashkes PJ, Berkun Y, Schalm S, Murias S, Dare JA, Brown D, et al. JAK1/2 inhibition with baricitinib in the treatment of autoinflammatory interferonopathies. J Clin Invest. 2018;128(7):3041-52.

21. Torrelo A. CANDLE syndrome as a paradigm of proteasome-related autoinflammation. Front Immunol. 2017:8:927.

22. Kim H, de Jesus AA, Brooks SR, Liu Y, Huang Y, VanTries R, Montealegre Sanchez GA, Rotman Y, Gadina M, Goldbach-Mansky R. Development of a validated interferon score using NanoString technology. J Interf Cytokine Res. 2018;38(4):171-85

23. Bohan A, Peter JB. Polymyositis and dermatomyositis (first of two parts). N Engl J Med. 1975:292(7):344-7.

24. Trieu EP, Targoff IN. Immunoprecipitation: Western blot for proteins of low abundance. Methods Mol Biol. 2015;1312:327-42.

25. Benjamini Y, Krieger AM, Yekutieli D. Adaptive linear step-up procedures that control the false discovery rate. Biometrika. 2006;93(3):491-507.

26. de Jesus AA, Hou Y, Brooks S, Malle L, Biancotto A, Huang Y, Calvo KR, Marrero B, Moir S, Oler AJ, et al. Distinct interferon signatures and cytokine patterns define additional systemic autoinflammatory diseases. J Clin Invest. 2020. https://doi.org/10.1172/JCl129301.

27. Rider LG, Aggarwal R, Machado PM, Hogrel JY, Reed AM, Christopher-Stine $L$, Ruperto N. Update on outcome assessment in myositis. Nat Rev Rheumatol. 2018;14(5):303-18.

28. Rice Gl, Melki I, Fremond ML, Briggs TA, Rodero MP, Kitabayashi N, Oojageer A, Bader-Meunier B, Belot A, Bodemer C, et al. Assessment of type I interferon signaling in pediatric inflammatory disease. J Clin Immunol. 2017; 37(2):123-32.

29. Li Y, Li C, Xue P, Zhong B, Mao AP, Ran Y, Chen H, Wang YY, Yang F, Shu HB. ISG56 is a negative-feedback regulator of virus-triggered signaling and cellular antiviral response. Proc Natl Acad Sci U S A. 2009;106(19): 7945-50.

30. Crowe WE, Bove KE, Levinson JE, Hilton PK. Clinical and pathogenetic implications of histopathology in childhood polydermatomyositis. Arthritis Rheum. 1982;25(2):126-39.

31. Gitiaux C, De Antonio M, Aouizerate J, Gherardi RK, Guilbert T, Barnerias C, Bodemer C, Brochard-Payet K, Quartier P, Musset L, et al. Vasculopathyrelated clinical and pathological features are associated with severe juvenile dermatomyositis. Rheumatology. 2016;55(3):470-9.

32. Gadina M, Johnson C, Schwartz D, Bonelli M, Hasni S, Kanno Y, Changelian P, Laurence A, O'Shea JJ. Translational and clinical advances in JAK-STAT biology: the present and future of jakinibs. J Leukoc Biol. 2018;104(3):499-514.

33. Fremond ML, Rodero MP, Jeremiah N, Belot A, Jeziorski E, Duffy D, Bessis D, Cros G, Rice Gl, Charbit B, et al. Efficacy of the Janus kinase 1/2 inhibitor ruxolitinib in the treatment of vasculopathy associated with TMEM173activating mutations in 3 children. J Allergy Clin Immunol. 2016;138(6):1752-5.

34. Aeschlimann FA, Fremond ML, Duffy D, Rice Gl, Charuel JL, Bondet V, Saire E, Neven B, Bodemer C, Balu L, et al. A child with severe juvenile dermatomyositis treated with ruxolitinib. Brain. 2018;141(11):e80.

35. Papadopoulou C, Hong Y, Omoyinmi E, Brogan PA, Eleftheriou D. Janus kinase $1 / 2$ inhibition with baricitinib in the treatment of juvenile dermatomyositis. Brain. 2019;142(3):e8.

36. Kato M, Ikeda K, Kageyama T, Kasuya T, Kumagai T, Furuya H, Furuta S, Tamachi T, Suto A, Suzuki K, et al. Successful treatment for refractory interstitial lung disease and pneumomediastinum with multidisciplinary therapy including tofacitinib in a patient with anti-MDA5 antibody-positive dermatomyositis. J Clin Rheumatol. 2019. https://doi.org/10.1097/RHU.0000000000000984.

37. Kurasawa K, Arai S, Namiki Y, Tanaka A, Takamura Y, Owada T, Arima M, Maezawa R. Tofacitinib for refractory interstitial lung diseases in antimelanoma differentiation-associated 5 gene antibody-positive dermatomyositis. Rheumatology. 2018;57(12):2114-9.

38. Sabbagh S, Almeida de Jesus A, Hwang S, Kuehn HS, Kim H, Jung L, Carrasco R, Rosenzweig S, Goldbach-Mansky R, Rider LG. Treatment of antiMDA5 autoantibody-positive juvenile dermatomyositis using tofacitinib. Brain. 2019;142(11):e59.

39. Reed AM, Crowson CS, Hein M, de Padilla CL, Olazagasti JM, Aggarwal R, Ascherman DP, Levesque MC, Oddis CV, Group RIMS. Biologic predictors of clinical improvement in rituximab-treated refractory myositis. BMC Musculoskelet Disord. 2015;16:257.

40. Nagaraju K, Ghimbovschi S, Rayavarapu S, Phadke A, Rider LG, Hoffman EP, Miller FW. Muscle myeloid type I interferon gene expression may predict therapeutic responses to rituximab in myositis patients. Rheumatology. 2016;55(9):1673-80.

41. Lopez De Padilla CM, Crowson CS, Hein MS, Strausbauch MA, Aggarwal R, Levesque MC, Ascherman DP, Oddis CV, Reed AM. Interferon-regulated chemokine score associated with improvement in disease activity in refractory myositis patients treated with rituximab. Clin Exp Rheumatol. 2015;33(5):655-63.

\section{Publisher's Note}

Springer Nature remains neutral with regard to jurisdictional claims in published maps and institutional affiliations.

Ready to submit your research? Choose BMC and benefit from

- fast, convenient online submission

- thorough peer review by experienced researchers in your field

- rapid publication on acceptance

- support for research data, including large and complex data types

- gold Open Access which fosters wider collaboration and increased citations

- maximum visibility for your research: over $100 \mathrm{M}$ website views per year

At $\mathrm{BMC}$, research is always in progress.

Learn more biomedcentral.com/submission 